\title{
Designing and Evaluating Sustainable Supply Chains: A Carbon Market Oriented Approach
}

\author{
Amin Chaabane, Amar Ramudhin, Marc Paquet \\ École de Technologie Supérieure \\ Department of Automated Manufacturing Engineering \\ 1100 Notre Dame Ouest, Montreal, Quebec, Canada, H3C 1 K3 \\ e-mail: \{amin.chaabane, amar.ramudhin, marc.paquet@etsmtl.ca\}
}

\begin{abstract}
Increasing regulatory legislations for carbon and waste management and the focus on corporate social responsibility are driving a major focus on supply chain sustainability. In this research, a goal programming model is proposed to address a supply chain design problem with environmental considerations. Carbon emissions (environmental dimension) and total logistics cost (economic dimension) are considered in order to evaluate the supply chain performance. A crucial contribution of our work is that, together with incorporating regulatory environmental constraints such as emissions "cap" and putting a price tag on carbon emissions, we comprehensively model compliance strategies for the supply chain including suppliers and sub-contractors selection, technology acquisition, and transportation modes configuration. The results obtained show that this approach is a viable decision tool and offer a good framework for designing and evaluating efficient and environmental supply chains.
\end{abstract}

Keywords: Sustainable Supply Chain Management, Carbon Emissions, Multiple Objective Programming

\section{INTRODUCTION}

Sulphur dioxide caps for electric utilities in the United States, carbon dioxide caps for companies across the European Union, and regulatory frameworks for greenhouse gases (GHGs) reduction in Canada and Australia are only a few of different regulations on air emissions that exist today. Corporations are realizing that GHGs reduction strategies and sustainability policies are bottom-line issues. As a consequence, firms need to consider several options and mechanisms in order to meet their legal obligation. Ideally, firms will reduce their emissions through sustainable actions such as the implementation of energy efficiency measures, the deployment of carbon capture and storage systems, or investing in other emissions reduction technologies. Also, companies can have access to other compliance mechanisms to earn carbon credits with the contribution to climate change technology fund or through an Emission Trading Scheme (ETS). ETS is based on a "cap-and-trade" approach where GHGs emissions cap is enforced. Companies that reduce emissions below the cap would be allocated tradable credits. Corporations with GHGs greater than the cap need to buy an equivalent amount of carbon credits to meet their regulatory obligation. There are already a range of active carbon trading programs to manage GHGs emissions. These programs establish a market by setting a target ("absolute cap" or "intensity target") and allow mandated participants to trade emissions allowances in order to meet compliance requirements at the lowest possible cost. The trading of emissions under a "cap-and-trade" system places supply chains managers in a different situation compared with the traditional control approach. First, corporations must consider the available options internally that might reduce GHGs and meet the cap. Second, they must compare the cost of implementing some of these options with the current trading price of emissions. Indeed, the trading system pushes all participants to compete in order to meet the "reduction target" at the minimum cost. At this level, the theory of a "cap-and-trade" emissions reduction system is extremely simple: it is a choice between "make or buy", either they make the reduction or they buy credits from someone who has done more than the required by the cap. In practice, the implementation of such an approach by supply chain managers is more complex because of the many options available at all stages of the supply chain: product design options, process options, transportation options, and reverse logistics options (recycle, reuse, etc.). A comprehensive optimization framework that links internal strategic decisionmaking (i.e. supply chain design and optimization) with the available carbon market-based mechanisms (i.e. emission trading) will be very useful to be compliant with environmental regulation and conserve the supply chain efficiency performance at the same time.

\section{PROBLEM STATEMENT AND RELATED WORK}

The government of Canada committed to develop and implement an integrated approach for industrial air emissions. The regulatory framework will mandate reductions of GHGs emissions for different industrial sectors. Thus, each corporation concerned with this regulation need to reduce emissions in efficient and cost-effective manner. To provide flexibility in achieving emissions caps and minimize the economic impact of the regulations, several options will be available. First, firms can reduce their own emissions through abatement actions. Also, they can contribute to a technology fund, which would then act as a means of promoting the development, deployment, and diffusion of technologies that reduce emissions of GHGs. In addition, 
they can use emissions trading, including inter-firms trading, emissions reduction credits from non regulated activities, and certain credits from the Kyoto Protocol's mechanisms.

Thus, an increasing number of companies across the country are struggling to evaluate the best strategic decisions in order to maintain an efficient and optimized supply chain under these new regulations. The integration of sustainability considerations is a key element in this context. For a supply chain, the objective of economic sustainability is to minimize the total logistic cost or maximize the profit of the different supply chain activities (purchasing, production, warehousing, distribution, recycling, etc). Regarding the environmental supply chain sustainability, permanent environmental damages should not be allowed. In this case, constraints related to efficient energy use, minimize waste (liquid and solid), and air pollution reduction constraints should be added. Therefore, the development of integrated analytical and numerical modeling approaches in order to study managerial decisions at the interface between the supply chain design and the environment are with great importance.

There are a number of approaches that strive to address different aspects of sustainable supply chain management. Recent papers (Srivastava, 2007; Seuring and Muller, 2008) present a good review of different elements related to supply chain sustainability: green design (Hugo and Pistikopoulos, 2005), inventory management (Ferretti et al, 2007), production planning and control for remanufacturing (Luo et al, 2001), green manufacturing, product recovery (Jayaraman, 1999) reverse logistics (Sheu et al, 2005), waste management (Ferretti et al, 2007), energy use (Dotoli et al, 2005), and GHGs emissions reduction (Ferretti et al, 2007). It is not surprising to see that mathematical modeling based methodologies are the most commonly used. Indeed, these models can be embedded as decision support systems (DSS). DSS proved their efficiency to manage traditional supply chain networks known today as advanced planning and scheduling systems (APS). We believe that analytical methods will continue to contribute also for Sustainable Supply Chain Management (SSCM). Moreover, sustainable supply chain practices require that supply chain managers take different decisions (strategic, tactical, and operational) while considering the balance (trade-offs) between the different sustainable objectives. These performances are usually conflicting and need advanced optimization techniques to find the best tradeoffs. Multi-Objective Optimization (MOO), a well established area within the field of operational research and being able to consider conflicting objectives (Cohon, 1978), enables modeling of many problems in business, engineering, operations management and also SSCM problems. Although the detailed literature about sustainability in supply chains, an explicit consideration and incorporation of environmental costs within supply chain activities is completely absent. Moreover, putting a price tag into carbon emissions is becoming critical for corporations. A good argument of this evidence is the carbon price in the European Union Emission Trading Scheme (EU ETS) which reaches $25 €$ in 2008 . Therefore, a critical contribution of this work is that, together with incorporating regulatory environmental constraints such as emissions limit (cap) and emissions cost/profit (price tag for carbon emission), we comprehensively model compliance strategies for supply chains, including suppliers and subcontractors selection, technology acquisition, and transportation modes configuration. Our modeling approach gives an efficient methodology for practitioners whom are beginning to deal with complex tradeoffs in complying with environmental regulations at various stages in the supply chain. Our work demonstrates how environmental regulations and supply chain's compliance strategies can be modeled using the classical operations research tool of mathematical programming. Finally, this approach can facilitate decisionsmaking through an understanding how the supply chain would optimally react to various forms and combinations of environmental regulations.

\section{MODEL OVERVIEW}

The aim of this model is to present a generic mathematical model to design sustainable supply chains that operate under emissions regulations. The model includes some general aspects of the traditional supply chain design problem combined with a novel business aspect: the environmental dimension. Environmental regulations are integrated in the form of "Emission limit" (or cap). To be in compliance with regulations, we allow a wider choice of options and managerial strategies as illustrated in Fig.1. Specifically, carbon trading mechanisms are integrated within the supply chain network design phase. Supply chain mangers have to decide between making some efforts internally through the reconfiguration of their supply chain in order to minimize their carbon footprint or to buy credits (or allowances) to be in compliance with the regulation.

The decision-making process is formulated as a mixed integer linear optimization program to decide on the supply chain configuration. The solution methodology first evaluates the cost of GHGs reduction of different strategic decisions (suppliers' and subcontractors' selection, product allocation, capacity utilization, transportation configuration), and compare the cost of implementing some of these options with the current trading price of emissions. This new formulation provides decision makers with the ability to understand the tradeoffs between total logistics costs and the impact of GHGs reduction. It also allows the offsetting of the latter ("carbon zero") through an interaction with the carbon market place. Moreover, the model is very useful for firms who are not concerned directly by the regulation of GHGs cap. Indeed, these firms with a GHGs level less than the cap can evaluate the opportunity behind a voluntary GHGs reduction action and the profit that they might make with selling carbon credits to the market place. The proposed approach is generic enough to be applied to any manufacturing context. In addition, we assume that the supply chain operate under a centralized system where a global coordinator is responsible for orchestrating the supply chain effort towards sustainability. A multi-objective optimization solution methodology is used to capture the trade-off between the economic and the environmental dimensions and evaluate their relative performance. 


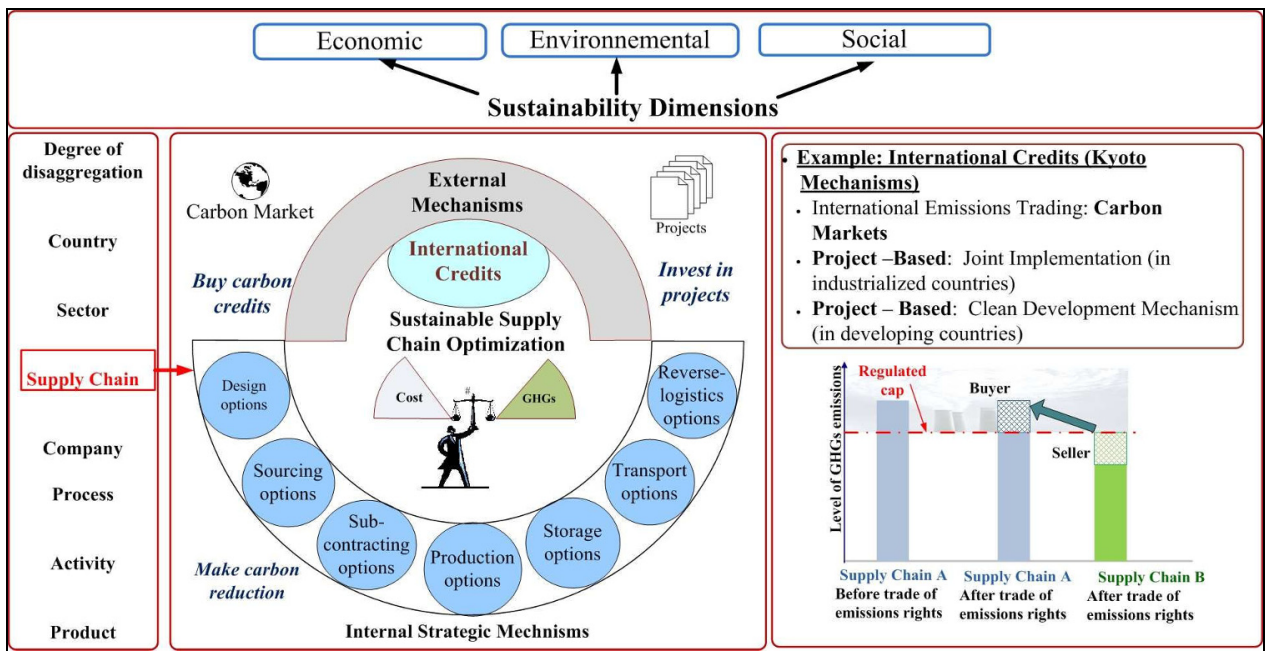

Fig. 1. Sustainable Supply Chain Design Methodology

\section{MODEL FORMULATION}

The model formulation presented in this paper extends a previous research work proposed by Ramudhin et al. (2008). A multi-level supply chain (see Fig. 2) composed of different potential suppliers, sub-contractors and plants that serve customers in different regions is considered. Different technologies can be acquired to manufacture products. Also, different transportation modes can be used for products delivery between nodes.

$P \quad$ Set of all products

$R \subset P \quad$ Set of raw materials

$M \subset P \quad$ Set of manufactured products

$C \subset M$ Set of finished products

$N \quad$ Set of all nodes

$G \quad$ Set of manufacturing technologies

$D \subset N \quad$ Set of customer zones

$S \subset N \quad$ Set of all subcontractors

$S_{p} \subset S \quad$ Set of subcontractors of product $p \in M$

$V \subset N \quad$ Set of suppliers of raw materials

$V_{p} \subset V$ Set of suppliers of raw material $p \in R$

$\mathrm{P}_{\mathrm{p}}^{\mathrm{s}} \quad$ Set of immediate successors of product $p \in P / C$

$\mathrm{SP}_{\mathrm{p}}^{\mathrm{s}} \quad$ Set of subcontractors for the set of immediate successors of product $p \in P / C$

$M_{i} \quad$ Set of products that can be manufactured by subcontractor $i \in S$

$R_{i} \quad$ Set of raw materials that can be supplied by supplier $i \in V$

$K \quad$ Set of all transportation modes $k \in K$

In this study, the following sets and indices are used:

The mathematical model requires the following parameters:

$\lambda_{i} \quad$ Fixed cost associated with the use of site $i \in S \cup V$

$\kappa^{g} \quad$ Fixed cost associated with the acquisition of

$\boldsymbol{\kappa}_{i}^{g} \quad$ technology $g \in G$ at site $i \in S$

The start-up cost associated with manufacturing

$a_{i p} \quad$ product $p \in M$ at site $i \in S_{p}$ $b_{i p} \quad$ Purchasing cost of raw material $p \in R$ at site $i \in V_{p}$

Unit cost of producing product $p \in M$ at site

$c_{i p}^{g} \quad i \in S_{p}$ using technology $g \in G$

transportation unit cost of product $p \in P$ from node

$t_{i j p}^{k} \quad i \in V_{p} \cup S_{p}$ to node $j \in S P_{p}^{S} \cup D$ using transportation mode $k \in K$

$l^{k} \quad$ Cost of a single shipment between nodes $i \in V \cup S$

$l_{i j}^{k} \quad$ and $j \in S \cup D$ using transportation mode $k \in K$

$\phi \quad$ Price per metric ton of carbon dioxide equivalent Greenhouse gases emissions factor per weight unit

$\alpha^{k} \quad$ and per distance unit due to the use of transportation mode $k \in K$ per ton-mile

Greenhouse gases emission factor (tones) per

$\beta_{i p}^{g} \quad$ weight of produced quantity of product $p \in M$ using the technology $g \in G$ at node $i \in S_{p}$

Cap Limit of emission fixed by regulation

$\theta_{p p^{\prime}} \quad$ Number of products $p \in P / C$ required to manufacture one unit of product $p^{\prime} \in P_{p}^{s}$

Maximum number of sites that can be opened for

$m_{p} \quad$ product $p \in M \cup R$

$e_{i p}$

Capacity of node $i \in S_{p}$ for product $p \in R$ (supplier's capacity)

Available time of at node $i \in S$ when using

$f_{i}^{g} \quad$ technology $g \in G$

Processing time on product $p \in M$ at node

$t e_{i p}^{g} \quad i \in S_{p}$ using technology $g \in G$

$d_{p d} \quad$ Number of product $p \in C$ required by demand node $d \in D$

Lower bound (in \%) on the aggregated capacity to

$\rho_{i} \quad$ be used if manufacturer or supplier $i \in S \cup V$ is chosen

$T \quad$ Total time available at the assembly line of subcontractor $i \in S$ 
$\tau_{i j} \quad$ Maximum number of transportation modes that can

$\tau_{i j} \quad$ be used between nodes $i \in V \cup S$ and $j \in S \cup D$

$\kappa^{k} \quad$ Volume capacity of transportation mode $k \in K$

$\psi^{k} \quad$ Weight capacity of transportation mode $k \in K$

$\pi_{p} \quad$ Weight of product $p \in P$

$\delta_{p} \quad$ Volume of product $p \in P$

$d(i, j) \quad$ Distance between nodes $i \in V \cup S$ and $j \in S \cup D$

To find the optimal configuration of the network, the following decision variables are required:

$A_{i} \quad$ Binary variable equals 1 if node $i \in V \cup S$ is open and operational for at least one product and 0 otherwise

$W_{i}^{g} \quad$ Binary variable equals 1 if technology $g \in G$ is selected at node $i \in S$

$Y_{i p} \quad$ Binary variable equals 1 if raw material $p \in R \cup M$ is assigned to node $i \in V_{p} \cup S_{p}$ and 0 otherwise

$X_{i p} \quad$ Number of units of product $p \in R$ supplied by node $i \in V_{p}$

$Q_{i p}^{g} \quad$ Number of units of product $p \in M$ manufactured by node $i \in S_{p}$ using technology $g \in G$

$F_{i j p}^{k} \quad$ Number of units of product $p \in P$ shipped from node $i \in V_{p} \cup S_{p}$ to node $j \in S P_{p}^{s} \cup D$ using transportation mode $k \in K$

$U^{k} \quad$ Number of shipments between nodes $i \in V \cup S$

$i j \quad$ and $j \in S \cup D$ using transportation mode $k \in K$

$Z_{i j}^{k} \quad$ Binary variable equals 1 if transportation mode $k \in K$ is used between nodes $i \in V \cup S$ and $j \in S \cup D$ and 0 otherwise

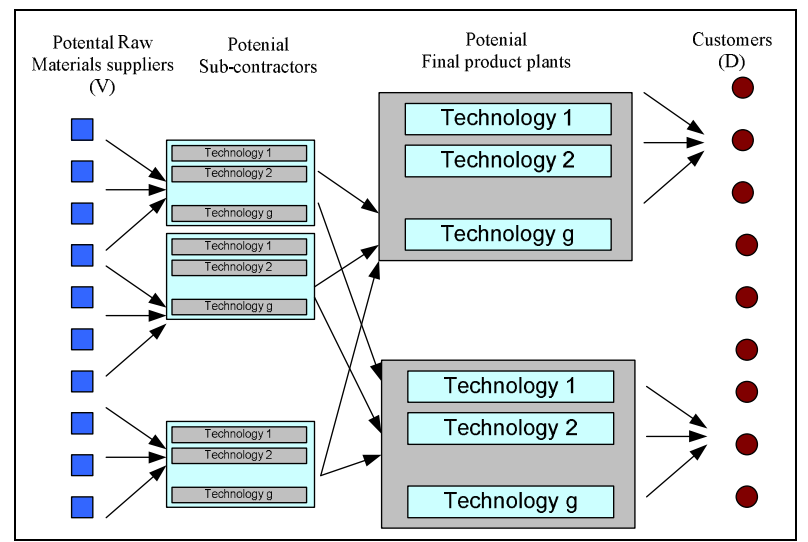

Fig. 2. Supply Chain Structure

In the following, two objective functions are considered. In the one hand, the objective of economic sustainability is to minimize the total logistics cost $\left(\mathbf{F}_{\mathbf{1}}\right)$ of the supply chain. It includes fixed, variable, and emission costs. On the other hand, the objective of environmental sustainability is to minimize the total emissions quantity of GHGs $\left(\mathbf{F}_{2}\right)$ calculated in term of tons of carbon dioxide equivalent $\left(\mathrm{tCO}_{2} \mathrm{e}\right)$.
Table 1: Cost structure of the objective function $\left(\mathbf{F}_{1}\right)$

Cost structure

Mathematical formulation

a) Cost for facilities $\sum_{i \in V \cup S} \lambda_{i} A_{i}$

b) Product assignment $\sum_{p \in R \cup M} \sum_{i \in V_{p} \cup S_{p}} a_{i p} Y_{i p}$

c) Technology acquisition $\sum_{i \in S} \sum_{g \in G} \kappa_{i}^{g} W_{i}^{g}$

d) Transportation lanes $\sum_{i \in S \cup V} \sum_{j \in S \cup D} \sum_{k \in K} l_{i j}^{k} U_{i j}^{k}$

e) Raw materials cost $\sum_{p \in R} \sum_{i \in V_{p}} b_{i p} X_{i p}$

f) Manufacturing cost $\sum_{p \in R} \sum_{i \in S_{p}} \sum_{g \in G_{p}} c_{i p}^{g} Q_{i p}^{g}$

(g) Transportation cost $\sum_{p \in M \cup R} \sum_{i \in S_{p} \cup V_{p}} \sum_{j \in S P_{p}^{s} \cup D} \sum_{k \in K} t_{i j p}^{k} F_{i j p}^{k}$

The GHGs emissions cost/profit (h) is calculated based on the credits compared to the limit of emissions ( Cap ) fixed by regulations using the following formula:

$$
\phi\left(\begin{array}{c}
\underbrace{\sum_{p \in M \cup R i \in S_{p} \cup V_{p}} \sum_{j \in S P} \cup \sum_{p \in K} \alpha^{k} \pi_{p} d(i, j) F_{i j p}^{k}}_{\text {GHGs Emissions from transportation }} \\
+\underbrace{\sum_{p \in M} \sum_{i \in S_{p}} \sum_{g \in G_{p}} \beta_{i p}^{g} \pi_{p} Q_{i p}^{g}}_{\text {GHGs Emissions from process }}-C a p
\end{array}\right)
$$

Therefore, the objective function $\mathbf{F}_{1}$ that represents the total operational cost to be minimized is:

Min $\mathbf{F}_{1}=(a)+(b)+(c)+(d)+(e)+(f)+(g)+(h)$

The objective function $\mathbf{F}_{2}$ that represents the total quantity of GHGs is as following:

$$
\begin{array}{r}
\operatorname{Min} \mathbf{F}_{2}=\underbrace{\sum_{p \in M \cup R} \sum_{i \in S_{p} \cup V_{p}} \sum_{j \in S P_{p}^{s} \cup D} \sum_{k \in K} \alpha^{k} \pi_{p} d(i, j) F_{i j p}^{k}}_{\text {GHGs Emissions from transportation }} \\
+\underbrace{\sum_{p \in M} \sum_{i \in S_{p}} \sum_{g \in G_{p}} \beta_{i p}^{g} \pi_{p} Q_{i p}^{g}}_{\text {GHGs Emissions from manufacturing }}
\end{array}
$$

For the supply chain network design model, there are many constraints to be considered. These constraints are of many kinds including the balance constraints of all products, the capacity limit constraints, the minimum capacity occupation constraints, and the demand satisfaction constraint. These elements are discussed below.

For each raw material and for each manufactured product, the number of operational sites should not exceed the maximum number allowed of suppliers and subcontractors:

$$
\sum_{i \in S_{p} \cup V_{p}} Y_{i p} \leq m_{p}(\forall p \in R \cup M)
$$

If a product (raw material) is assigned to a node (supplier), then the number of products supplied by this supplier must not exceed its capacity for this product:

$$
X_{i p}-e_{i p} Y_{i p} \leq 0 \quad\left(\forall p \in R, \forall i \in V_{p}\right)
$$


A product (semi-finished or final product) is manufactured in a node (subcontractor) only if the product is assigned to this node:

$$
\sum_{g \in G} Q_{i p}^{g}-M Y_{i p} \leq 0 \quad\left(\forall p \in M, \forall i \in S_{p}\right)
$$

Then the overall processing time used must not exceed the total available time at its assembly line or manufacturing facility:

$$
\sum_{p \in M_{i}} t e_{i p}^{g} Q_{i p}^{g}-f_{i}^{g} W_{i}^{g} \leq 0 \quad(\forall i \in S, \forall g \in G)
$$

There is usually a minimum amount of the aggregate capacity of a subcontractor that should be consumed to justify the establishment of a contract. This consideration leads to constraints (6) where the first term is the total time used at the assembly line or manufacturing facility of subcontractor $i$ in order to manufacture all the products. The second term of the left hand side of the inequality is the minimum time to be used if subcontractor $i$ is chosen:

$$
\sum_{p \in M_{i}} \sum_{g \in G_{p}} t e_{i p}^{g} Q_{i p}^{g}-\rho_{i} \sum_{g \in G} f_{i}^{g} W_{i}^{g} \geq 0, \forall i \in S
$$

To make a deal with a supplier, the minimum capacity can also be considered. Here, the minimum capacity to be used is a percentage of the total weight of all maximum quantities of raw materials that can be supplied by the supplier:

$$
\sum_{p \in R_{i}} X_{i p}-\left(\rho_{i} \sum_{p \in R_{i}} b_{i p}\right) A_{i} \geq 0(\forall i \in V)
$$

The constraints of flow out of suppliers' nodes are given by the equalities below:

$$
X_{i p}-\sum_{j \in S P_{p}^{s} \cup D} \sum_{k \in K} F_{i j p}^{k}=0 \quad\left(\forall p \in P, \forall i \in V_{p}\right)
$$

The constraints of flow out of subcontractors' nodes are given by the equalities below:

$$
\sum_{g \in G_{p}} Q_{i p}^{g}-\sum_{j \in S P_{p}^{s} \cup D} \sum_{k \in K} F_{i j p}^{k}=0 \quad\left(\forall p \in P, \forall i \in S_{p}\right)
$$

For each product, the quantity that arrives to a node must equal the quantity needed to manufacture next higher assemblies:

$$
\sum_{j \in S_{p}} \sum_{k \in K} F_{j i p}^{k}-\sum_{p^{\prime} \in P_{p}^{s}} \sum_{g \in G_{p^{\prime}}} \theta_{p p^{\prime}} Q_{i p^{\prime}}^{g}=0 \quad\left(\forall p \in M, \forall i \in S P_{p}^{s}\right)
$$

The quantity of finished products shipped from all its subcontractors to the demand node must equal the demand of that product:

$$
\sum_{i \in S_{p}} \sum_{k \in K} F_{i d p}^{k}=d_{p d}(\forall p \in C, \forall d \in D)
$$

For each couple of nodes, there is a maximum number of transportation modes that can be used.

$$
\sum_{k \in K} Z_{i j}^{k} \leq \tau_{i j} \quad(\forall i \in V \cup S, \forall j \in S \cup D)
$$

The quantity of products shipped between two nodes is limited by the capacity of transportation mode and the number of shipments. While the first set of constraints (12) expresses the volume capacity and the second set (13) expresses the weight capacity:

$$
\begin{gathered}
\sum_{p \in R_{i} \cup M_{i}} \delta_{p} F_{i j p}^{k}-\kappa^{k} U_{i j}^{k} \leq 0 \quad(\forall i \in V \cup S, \forall j \in S \cup D, \forall k \in K) \\
\sum_{p \in R_{i} \cup M_{i}} \pi_{p} F_{i j p}^{k}-\psi^{k} U_{i j}^{k} \leq 0 \quad(\forall i \in V \cup S, \forall j \in S \cup D, \forall k \in K)
\end{gathered}
$$

The following are logical constraints. The number of shipments between two nodes for a given transportation mode is not nil only if the transportation mode is actually used. This yields to the following constraints:

$$
U_{i j}^{k}-\theta Z_{i j}^{k} \leq 0 \quad(\forall i \in V \cup S, \forall j \in S \cup D, \forall k \in K),
$$

$\theta$ is a big number

A site is selected if it is open for one product at least:

$$
Y_{i p}-A_{i} \leq 0 \quad\left(\forall i \in S \cup V, \forall p \in M_{i} \cup R_{i}\right)
$$

In the case of minimizing $\mathbf{F}_{2}$, the following constraints should be added to the model. No assignment of raw material to supplier if the raw material is not supplied by this supplier:

$$
Y_{i p}-X_{i p} \leq 0 \quad\left(\forall P \in P, \forall i \in V_{p}\right)
$$

No assignment of manufactured product to plants if the product is not manufactured in this plant

$$
Y_{i p}-\sum_{g} Q_{i p}^{g} \leq 0 \quad\left(\forall P \in P, \forall i \in V_{p}\right)
$$

A technology is acquired only if it used to produce at least one product:

$$
W_{i}^{g}-\sum_{p \in M_{i}} Q_{i p}^{g} \leq 0 \quad(\forall i \in S, \forall g \in G)
$$

A site is selected if it is open for one product at least:

$$
A_{i}-\sum_{p \in M_{i} \cup R_{i}} Y_{i p} \leq 0 \quad(\forall i \in S \cup V)
$$

\section{CASE STUDY}

In this study, we consider the case of a firm with high level of GHGs emissions and subject to a new regulation that caps carbon emissions. The different products are aggregated into on product family with two semi finished products that are manufactured with four parts sourced from various external suppliers. Different potential technologies are available to produces products. Three freight transportation modes are considered: rail, air, and road to ship products between supply chain nodes. GHGs are limited to carbon dioxide $\left(\mathrm{CO}_{2}\right)$ emissions caused by production and transportation activities. Emissions factors for the three transportation modes $\left(\alpha^{k}\right)$ considered in this example are based on the recent accurate study published in Facanha and Horvath (2007). The manufactured products are primarily composed of steel materials and the emission factors $\left(\beta_{i p}^{g}\right)$ for different technologies is obtained form the IPCC Emission Factor Data Base [e.g. $\beta_{i p}^{g}=1.6$ tons $\mathrm{CO}_{2} /$ ton steel product].

The model is first solved by CPLEX Interactive Optimizer 10.0 considering only objective function $\mathbf{F}_{\mathbf{1}}$ (scenario 1 ). The optimal cost is $2696051 \$$. The total emission quantity relative to this solution is $21067 \mathrm{tCO}_{2} \mathrm{e}$. In addition, the model is solved while considering only objective function $\mathbf{F}_{\mathbf{2}}$ (scenario 2). The optimal GHGs emissions quantity is 19988 $\mathrm{tCO}_{2} \mathrm{e}$. The total cost relative to this solution is $2864915 \$$. Fig. 2 shows that the total logistics cost decreases with each increase in the upper bound of $\mathrm{CO}_{2} \mathrm{e}$ emissions $(\mathrm{UB})$ ) as the model seeks less costly solution alternatives which have higher emission rates. It stabilizes after a while. The selection of low cost technologies was observed. From a managerial perspective, this means that those companies might have to look for new sourcing, production or transportation 
alternatives and invest in environmentally friendly technologies in order to reduce GHGs emissions. In this example, GHGs emissions are more than the cap for the both scenarios, and there is a need to buy carbon credits.

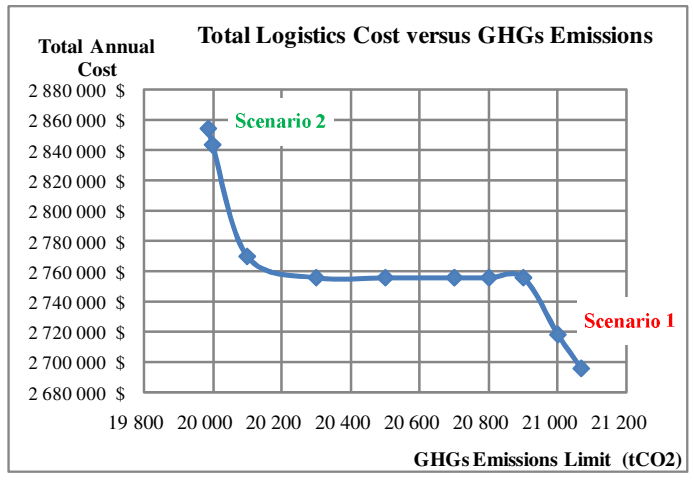

Fig. 3. Total logistics Cost versus Carbon Emissions

Fig. 3 also shows that the total logistics cost $\left(\mathrm{F}_{1}\right)$ and carbon emissions $\left(\mathrm{F}_{2}\right)$ are two conflicting objectives. Thus, the application of a multi-objective optimization procedure could help to determine the best trade-off. The goal programming (GP), a very well-known multi-objective solution procedure, was used for the previous example in order to find the tradeoffs between the total logistics cost and GHGs emissions. The GP solution has an emission quantity of 20302 tCO2e for an operational cost of $\$ 2718302$. The total logistic cost for the GP solution is only $3.5 \%$ greater than the efficient scenario and $0.9 \%$ greater than the green scenario in terms of emission quantity (compared to $6.3 \%$ increase in total logistic cost for the green scenario and $5.1 \%$ increase in carbon emissions for the efficient scenario). Clearly, this example demonstrates that by using a multi-objective approach, it is possible to achieve trade-offs with a good reduction in GHGs emissions while maintaining operational costs under control (Fig. 4).

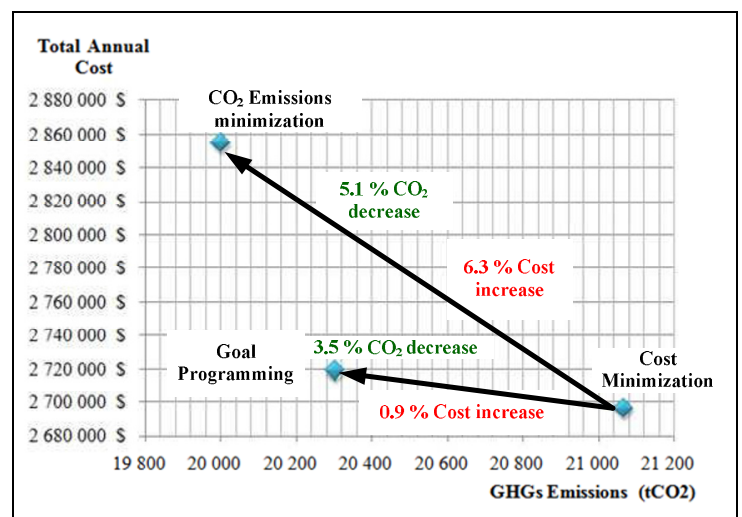

Fig. 4. Compliance strategies

This case study demonstrates that the proposed decision model has the potential to be a tool for designing and evaluating sustainable supply chains under the Emission Trading Scheme. The evaluation of the economic impact of regulation into the supply chain is identified with much more accuracy. Decisions are made now with much more visibility. A carbon market sensitive strategy allows the evaluation of carbon credits to buy and thus a more accurate estimation of "Emission Cost".

\section{CONCLUSIONS}

The main contribution of this paper is the development of an integrated model for sustainable supply chain network design leveraging the opportunities offered by carbon trading markets. This work enriches clearly the literature on designing and evaluating the performance of sustainable supply chains. The explicit consideration and incorporation of environmental cost (emissions cost) is so relevant in this context. Using the proposed model, supply chain managers are now able to determine the GHGs footprint of supply chains operations. They can determine if they qualify for carbon credits or must purchase credits on the carbon market place. That will help them decide on the best reconfiguration strategy for their supply chain to be in compliance with new environmental regulations. Moreover, different research questions can be answered using this model, but the most important of them is how carbon prices will impact supply chain operations and the competitive strategy of a company in the future?

\section{REFERENCES}

Cohon, J. L. (1978). Multiobjective programming and planning. New York: Academic Press.

Dotoli, M., Fanti, M. P., Meloni, C., \& Zhou, M. C. (2005). A multi-level approach for network design of integrated supply chains. International Journal of Production Research, 43(20), 4267-4287.

Ferretti, I., Zanoni, S., Zavanella, L., \& Diana, A. (2007). Greening the aluminium supply chain. International Journal of Production Economics, 108(1-2), 236-245.

Facanha, C., \& Horvath, A. (2007). Evaluation of Life-Cycle Air Emission Factors of Freight Transportation. Environmental Science \& Technology, 41(20), 7138-7144.

Hugo, A., \& Pistikopoulos, E. N. (2005). Environmentally conscious long-range planning and design of supply chain networks. Journal of Cleaner Production: Recent advances in industrial process optimisation, 13(15), 1471-1491.

Jayaraman, V., Guide, V. D. R., Jr., \& Srivastava, R. (1999). Closed-loop logistics model for remanufacturing. Journal of the Operational Research Society, 50(5), 497-508.

Luo, Y., Zhou, M., \& Caudill, R. J. (2001). An integrated Esupply chain model for agile and environmentally conscious manufacturing. IEEE/ASME Transactions on Mechatronics, 6(4), 377-386.

Point Carbon (2008). www.pointcarbon.com

Ramudhin. A., A. Chaabane, M. Kharoune, and M. Paquet, "Carbon Market Sensitive Green Supply Chain Network Design". In IEEE International Conference on,Industrial Engineering and Engineering Management, 2008, pp. 10931097.

Srivastava, S. K. (2007). Green supply-chain management: A state-of-the-art literature review. International Journal of Management Reviews, 9(1), 53-80.

Seuring, S., \& Muller, M. (2008). From a literature review to a conceptual framework for sustainable supply chain management. Journal of Cleaner Production, 16(15), 16991710.

Sheu, J.-B., Chou, Y.-H., \& Hu, C.-C. (2005). An integrated logistics operational model for green-supply chain management. Transportation Research Part E: Logistics and Transportation Review, 41(4), 287-313. 